\title{
Aborto provocado e violência doméstica entre mulheres atendidas em uma maternidade pública de Salvador-BA
}

\author{
Voluntary abortion and domestic violence among women attended at a public maternity hospital of Salvador-BA
} Aborto provocado y violencia doméstica entre mujeres asistidas en una maternidad pública de Salvador-BA

Normélia Maria Freire Diniz', Solange Maria dos Anjos Gesteira', Regina Lúcia Mendonça Lopes", Rosana Santos Mota"I', Bárbara Angélica Gómez Pérez ${ }^{\mathrm{IV}}$, Nadirlene Pereira Gomes ${ }^{\mathrm{V}}$

\footnotetext{
' Universidade Federal da Bahia, Escola de Enfermagem, Grupo de Pesquisa Violência, Saúde e Qualidade de Vida, e Grupo de Estudos sobre Saúde da Mulher, linha de pesquisa Mulher, Violência e Saúde. Salvador-BA, Brasil.

"Universidade Federal da Bahia, Escola de Enfermagem, Grupo de Pesquisa Violência, Saúde e Qualidade de Vida. Salvador-BA, Brasil.

I" Universidade Federal da Bahia, Escola de Enfermagem, Programa de Pós-Graduação em Enfermagem (Mestranda), Grupo de Pesquisa Violência, Saúde e Qualidade de Vida. Salvador-BA, Brasil.

Iv Faculdade Jorge Amado. Universidade Federal da Bahia, Escola de Enfermagem, Grupo de Pesquisa Violência, Saúde e Qualidade de Vida. Salvador-BA, Brasil.

${ }^{v}$ Universidade Federal do Vale do São Francisco. Universidade Federal da Bahia, Escola de Enfermagem, Grupo de Pesquisa Violência, Saúde e Qualidade de Vida. Salvador-BA, Brasil.
}

Submissão: 06-05-2010 Aprovação: 27-01-2012

\section{RESUMO}

Estudo quantitativo, com o objetivo de estudar a violência doméstica em mulheres em situação de aborto provocado. Foram entrevistas 147 mulheres internadas por aborto provocado numa maternidade pública, na cidade de Salvador-BA. Os sujeitos foram, na maioria, mulheres jovens, negras, com baixa escolaridade, dependentes economicamente dos cônjuges, que vivenciam violência psicológica, física e sexual cometida pelos cônjuges. Quase metade das mulheres vivenciou violência doméstica durante a gravidez atual, sendo este o motivo do aborto para $67 \%$ delas. Conclui-se que existe uma associação entre a vivência de violência doméstica e o aborto provocado. Isso repercute na saúde mental das mulheres, que desenvolvem sintomas do transtorno de estresse pós-traumático. Necessitase, portanto, de um olhar por parte dos profissionais de saúde de modo a identificar a violência doméstica enquanto agravo e associá-la ao aborto provocado, o que requer transformação no modelo de formação, incorporando a violência doméstica como objeto da saúde. Descritores: Enfermagem; Enfermagem obstétrica; Saúde da mulher; Violência doméstica; Aborto induzido.

\section{ABSTRACT}

Quantitative study in order to study domestic violence in women with induced abortion. Interviews were conducted with 147 women hospitalized for induced abortion in a public maternity hospital in Salvador, Bahia. The subjects are characterized by mostly women, black, poorly educated, economically dependent on spouses, experienced psychological abuse, physical and sexual abuse committed by their spouses. Almost half of the women were victims of domestic violence during the current pregnancy, and that was the reason for inducing abortion for $67 \%$ of them. The study reveals an association between experience of domestic violence and induced abortion. As mental health consequences, they developed symptoms of post trauma stress disorder. It is necessary that the health professionals consider the cues to identify domestic violence as a health problem associated with induced abortion, which requires a transformation on the training model, including domestic violence as a health issue.

Key words: Nursing; Obstetrical nursing; Women's health; Domestic violence; Induced abortion.

\section{RESUMEN}

Estudio cuantitativo, que tuvo como objetivo estudiar la violencia doméstica en mujeres con aborto inducido. Se realizaron entrevistas con 147 mujeres internadas por aborto inducido en una maternidad pública de Salvador-BA. Los sujetos se caracterizaran por ser, en su mayoría mujeres jóvenes, negras, con un bajo nivel educativo, dependientes económicamente de los conyugues, con experiencia de abusos psicológicos, físicos y sexuales cometidos por los cónyuges. Casi la mitad de las mujeres fueron víctimas de violencia doméstica durante el embarazo actual, siendo este el motivo de aborto para $67 \%$ de ellas. El estudio reveló una asociación entre la experiencia de violencia doméstica y el aborto inducido, con consecuencias para la salud mental de las mujeres, que desarrollan síntomas de trastorno de estrese post traumático. Es necesaria una mirada por parte de los profesionales de la salud para identificar la violencia doméstica como un problema de salud asociado con el aborto inducido, lo que requiere una transformación del modelo de formación, que incorpore la violencia doméstica como un tema de salud.

Palabras clave: Enfermaría obstétrica, Violencia domestica, Aborto inducido, Salud de la mujer. 


\section{INTRODUÇÃO}

No Brasil, mais de $30 \%$ das gestações terminam em aborto. As mulheres mais atingidas são as de baixa renda, particularmente as negras. Em 2004, cerca de 240 mil internações pelo SUS foram motivadas por curetagens pós-aborto ${ }^{(1)}$. A interrupção da gravidez representa a quinta maior causa de internações na rede pública de saúde brasileira e é a terceira causa de morte materna. Nas cidades do Nordeste do país, o aborto provocado é uma das mais significativas causas de morte materna. Em Salvador, desde o início da década de 90 do século XX, o aborto vem despontando como a primeira causa de óbito entre mulheres em idade fértil, sendo que, a cada mês, morre uma mulher vítima de aborto ${ }^{(2)}$.

O aborto provocado é uma das principais causas de morbi-mortalidade materna em países onde existem restrições legais ao aborto, especialmente quando realizado por pessoal não qualificado(3). As mulheres que não morrem podem ter complicações graves, tais como hemorragias, septicemia, peritonite e choque, e outras podem ter comprometimentos psicológicos.

A violência durante a gravidez se insere dentro da saúde reprodutiva, sendo considerada, mundialmente, uma questão de interesse particular. Alguns estudos apontam a gravidez como fator de risco para a ocorrência de violência doméstica, destacando-se, entre suas consequências, o abortamento e a natimortalidade ${ }^{(4)}$.

São muitas as formas que a violência doméstica assume: desde os maus-tratos, que deixam marcas no corpo da vítima e podem culminar com a morte, até as mais sutis, como as torturas psíquicas diárias, que aterrorizam e minam a autoestima. $\mathrm{O}$ Ministério da Saúde já reconhece em suas ações políticas que a violência contra a mulher tem impacto sobre a saúde física e mental das mulheres e sobre os membros da família, orientando para o fato de que o atendimento às pessoas em situação de violência deva contemplar o aspecto psicossocial da assistência, independente da opção de interromper ou não a gestação(5). Embora o aborto seja legal para os casos de estupro, sua prática não é aceita quando se trata de situações de violência sexual no âmbito conjugal, o que resulta no aborto provocado ilegal.

Diante do exposto, este estudo tem como objetivo geral estudar a violência doméstica em mulheres em situação de aborto provocado e como objetivos específicos caracterizar as mulheres em situação de aborto provocado segundo as condições sociais, demográficas e obstétricas e a história de violência doméstica; descrever as formas de violência sofrida por mulheres em situação de aborto provocado; e identificar as repercussões de violência doméstica para a saúde mental de mulheres em situação de aborto provocado.

\section{METODOLOGIA}

Trata-se de um estudo descritivo, exploratório, de natureza quantitativa. A população da pesquisa foi constituída por muIheres internadas em uma maternidade pública de Salvador-BA por aborto provocado. A seleção da amostra foi de conveniência e constou de todas as mulheres que provocaram aborto e que se encontrava em processo de alta hospitalar no período compreendido entre os meses de fevereiro e abril de 2006. Diariamente, foram consultados os prontuários para a identificação dos sujeitos, considerando o período mínimo de internação, que era em torno de $24 \mathrm{hs}$. A consulta nos prontuários e a entrevista foram realizadas por uma mestranda, enfermeira obstetra, e uma aluna da graduação em enfermagem, ambas treinadas em oficinas vinculadas a Projetos de Extensão, onde semanalmente, durante seis meses, se trabalhou a temática aborto provocado.

As mulheres foram informadas acerca do objetivo da pesquisa, de sua relevância social, da garantia ao sigilo das informações. E ainda do não pagamento pela participação, do anonimato, da publicação dos resultados, do tempo de armazenamento destas informações (cinco anos) e do direito de desistir do estudo a qualquer momento, não havendo, por isso, prejuízo no atendimento. Todos esses aspectos éticos estão baseados na Resolução 196/96 ${ }^{(6)}$ do Conselho Nacional de Saúde, que norteia a prática das pesquisas em seres humanos, e estão expressos no Termo de Consentimento Livre e Esclarecido o qual contém, ainda, o telefone para contato com as pesquisadoras e dados para contato com o Comitê de Ética em Pesquisa - SESAB (Registro CEP: CAAE - 0012.0.053.00006), que apreciou o projeto e aprovou seu desenvolvimento.

Visto que a amostra compreendeu todas as situações de aborto provocado no intervalo de tempo determinado, vale dizer que algumas das entrevistadas ainda não tinham completado 18 anos, o que as colocava no grupo de adolescentes, segundo definição do Estatuto da Criança e do Adolescente. Contudo, diante da relevância social do estudo, do fato de o aborto provocado ser uma realidade nesta faixa etária e de ocorrer em um contexto sigiloso, o CEP aprovou a realização do estudo sem restrições.

O contato com as mulheres se deu na enfermaria, no momento da alta hospitalar. Todas as mulheres aceitaram participar do estudo e foram convidadas a responder à entrevista em uma sala privada.

A consulta nos prontuários foi apenas para fins de identificação dos casos de aborto e condição de alta hospitalar. Utilizamos como técnica de coleta de dados a entrevista, acompanhada de um formulário semiestruturado com perguntas abertas e fechadas. Elegemos como variáveis condições sociais e demográficas (idade, cor, grau de escolaridade, estado civil, trabalho, dependência financeira), história obstétrica (gestações anteriores, abortamento provocado anteriormente), vivência de violência (algum momento da vida, na gestação atual), tipo de violência, tipo de agressor; motivos para o aborto; consequências do aborto para a saúde das mulheres; sintomas de estresse pós-traumático.

$\mathrm{Na}$ identificação das repercussões psicológicas do aborto para as mulheres, utilizamos a escala do Manual de Diagnósticos e Estatística de Transtornos Mentais (DSM IV), que permite mostrar sintomas do estresse ${ }^{(7)}$. Independentemente do grau de estresse, todas as mulheres com história de violência doméstica, atual ou anterior, foram orientadas quanto à importância do Centro de Referência Loreta Valadares, que compõe a rede para atendimento às mulheres em situação de violência e 
presta assistência especializada nos âmbitos psicológico, social e jurídico para as situações de violência doméstica.

\section{APRESENTAÇÃO E DISCUSSÃO DOS RESULTADOS}

\section{Caracterização dos sujeitos}

Foram entrevistadas 147 mulheres internadas por aborto provocado. O maior percentual se encontrava na faixa etária compreendida entre os 19 e os 24 anos $(45,5 \%)$, seguido de adolescentes na faixa etária compreendida entre os 12 e os 18 anos de idade $(22,5 \%)$. Resultados semelhantes foram encontrados nos estudos de Martins ${ }^{(8)}$ e Hardy et al. ${ }^{(9)}$. Destacamos a precocidade do ato sexual pois, neste estudo, encontramos histórias de abortamento em meninas com 12 anos.

Cerca de $90 \%$ das entrevistadas eram negras, autodefinindo-se como pretas $(54,5 \%)$ ou pardas $(36 \%)$. Este percentual reflete a situação da população de Salvador (BA), estimada em $83 \%$ de negros em $2004{ }^{(10)}$. No que tange à escolaridade, o estudo mostrou que as mulheres entrevistadas, na sua maioria, declararam possuir o $1^{\circ}$ grau incompleto $(38,8 \%)$; o $2^{\circ}$ grau incompleto mostrou ser o segundo maior índice $(33,3 \%)$. Corroborando o que foi encontrado no estudo, pesquisa sobre desigualdades raciais em uma metrópole brasileira aponta que o nível de escolaridade das mulheres de raça negra está entre o $1^{\circ}$ grau incompleto e o $2^{\circ}$ grau incompleto ${ }^{(11)}$.

Chama a atenção o grande número de adolescentes e adultas jovens negras, com baixa escolaridade, cuja gravidez se deu de forma espontânea. Esta realidade guarda relação com a vivência da sexualidade sem necessariamente observar o corpo biologicamente passível de gestar, elevando, assim, os índices de gravidez indesejada e, por conseguinte, o aborto provocado.

Quanto ao estado civil, 54,4\% das entrevistadas conviviam conjugalmente com o companheiro, embora apenas $8,2 \%$ destas se declarassem casadas e as demais, em união consensual. As solteiras representaram $42,8 \%$ das entrevistadas e as viúvas, $2,8 \%$. Percebe-se que as mulheres usufruem de sua sexualidade independentemente da situação de conjugalidade e que a interrupção da gravidez não tem relação com o fato de ser solteira ou casada. O estudo "Decisão de abortar" também demonstrou que tanto as mulheres em união estável como as solteiras realizam abortamento provocado ${ }^{(12)}$.

Com relação à variável trabalho, o estudo mostrou que cerca de $40 \%$ das entrevistadas se ocupavam com atividades domésticas, sem remuneração. Um percentual equivalente de mulheres $(43,6 \%)$ declarou exercer ocupações remuneradas, fora de casa, tais como empregada doméstica, manicure, babá, cuidadora de idosos. Nota-se que, embora exercendo atividades remuneradas fora de casa, as funções são eminentemente domésticas.

Dentre as entrevistadas, $80 \%$ se encontravam em relação de dependência financeira ( $47,9 \%$ eram parcialmente dependentes e $52,1 \%$, totalmente dependentes). Destas, $60 \%$ disseram receber ajuda do marido/companheiro. As demais recebiam ajuda dos familiares, principalmente de pai e mãe. Este alto percentual de dependência econômica não tem relação apenas com o grande número de mulheres desempregadas $(44 \%)$, mas também com aquelas que ocupam atividades remuneradas. Vale salientar que muitas destas ocupações constituem empregos informais, ou seja, subempregos, gerando uma renda mensal abaixo do salário mínimo. Além disso, as mulheres ainda são as responsáveis pelas tarefas domésticas e pelo cuidado com os filhos, o que implica uma dupla jornada de trabalho. Yannoulas ${ }^{(13)}$ mostra, em seu estudo, que grande parte das trabalhadoras, principalmente as negras, se concentra em setores que oferecem menores rendimentos e possibilidades de ascensão, tais como, por exemplo, os serviços domésticos.

Quanto às condições obstétricas, a maioria das mulheres $(54,4 \%)$ apresentou uma ou duas gestações anteriores. Igualmente expressivo foi o número de mulheres com três ou quatro gestações $(34,7 \%)$. Com relação ao aborto provocado, $64,0 \%$ das mulheres declararam ser aquele o primeiro aborto realizado. Estes dados são compatíveis com os resultados do estudo de Hardy et al. ${ }^{(9)}$.

Continuando, $31 \%$ das mulheres afirmaram ter realizado entre dois e três abortos e $5 \%$, de quatro a sete. Resumindo, nota-se que mais de um terço das entrevistadas já havia realizado pelo menos outro aborto, sinalizando para a dificuldade de associar a vivência da sexualidade e a possibilidade de uma gravidez, o que, por sua vez, compromete a prevenção.

As mulheres se referiram a complicações do abortamento para sua saúde, tais como, secreção vaginal fétida $(32,6 \%)$, hemorragia $(82,9 \%)$ e infecção $(29,3 \%)$, entre outras. As complicações do abortamento incompleto representam a quinta causa de internações no SUS e respondem por $9 \%$ dos óbitos maternos. A curetagem pós-aborto representa o segundo procedimento obstétrico mais realizado nos hospitais da rede pública $^{(14)}$. Por ser ilegal, as mulheres que abortam se encontram expostas a praticas não seguras, levando com mais frequência a situações de risco.

A situação econômica $(36,7 \%)$ foi o principal motivo alegado pelas mulheres para a prática do aborto, estando esta associada à falta de condições para criar o filho. Os demais motivos foram vivência de violência doméstica $(27,9 \%)$, pouca idade $(19 \%)$ e o fato de uma gravidez atrapalhar os planos para o futuro $(10 \%)$. Com relação aos planos futuros, não querer ter filhos na ocasião foi uma das razões mais citadas para a prática em estudo realizado por Martins et al. ${ }^{(8)}$.

\section{Vivência de violência doméstica}

O estudo mostrou que $88 \%$ das entrevistadas (130 mulheres) declararam vivência de violência doméstica em algum momento de suas vidas. Estudo realizado em uma maternidade pública mostrou que $80 \%$ das mulheres entrevistadas tinham história de violência doméstica ${ }^{(15)}$.

Vale ressaltar que, entre estas, $47 \%$ sofreram violência na gestação atual. Este percentual condiz com o que foi encontrado no estudo de Fernandes ${ }^{(16)}$, que apresenta um percentual de $50 \%$ de história de violência doméstica entre mulheres que provocaram aborto. Das mulheres que sofreram violência doméstica na gravidez atual, $67 \%$ disseram ter realizado o aborto em decorrência desta vivência. Os dados sugerem, portanto, uma associação entre a vivência de violência doméstica e o aborto provocado.

Os principais perpetradores da violência doméstica praticada contra as mulheres $(70 \%)$ possuem alguma relação de 
conjugalidade com as mesmas (maridos/companheiros, namorados ou ex-maridos). Isto condiz com os resultados encontrados em pesquisa de Menezes $^{(4)}$, que revela ser o marido/companheiro o principal praticante da violência contra a mulher. A violência praticada pelo parceiro íntimo constitui a forma mais endêmica de violência contra a mulher, sendo, muitas vezes, aceito culturalmente o direito do homem de dispor da mulher.

As mulheres citaram vivência de violência expressa sob as formas psicológica $(96,7 \%)$, física $(11,5 \%)$ e sexual $(6,5 \%)$. Para a violência psicológica, mais expressiva, foram comuns relatos referentes a humilhações, xingamentos, acusações (de ter amantes, por exemplo), violação do direito de ir e vir (foram impedidas de sair de casa, inclusive para trabalhar) e negligências (ficaram sem assistência durante o período da gestação ou quando estavam doentes). Independentemente da forma como se expresse, a vivência de violência doméstica, sobretudo durante a gravidez, leva a mulher a adoecer.

\section{Adoecimento}

O estudo mostra uma associação entre aborto provocado e o desenvolvimento de sintomas do Transtorno por Estresse Pós-Traumático (Tabela 1).

Com relação aos sintomas de imagens em flashback, que fazem com que as mulheres revivam o trauma e ao sentimento de culpa, o percentual entre as mulheres que não têm história de violência foi de 35,2\%. Já naquelas com história de violência e em vivência de violência na gestação atual, este percentual aumenta consideravelmente $(66,6 \%$ e $83,6 \%$, respectivamente).

Quanto a julgar-se a si mesmas e sentir culpa, quanto também aos sentimentos de desamparo e impotência, eles se fizeram presentes em mulheres sem história de violência $(41,1 \%$ e $11,7 \%$, respectivamente), é verdade, mas de forma menos acentuada que em mulheres com história de violência $(46,3 \%$ e $30,4 \%$, respectivamente) e em vivência de violência na gestação atual $(83,6 \%$ e $59 \%)$.

Continuando, vimos que $17,6 \%$ e $29,4 \%$ das mulheres sem história de violência apresentaram distúrbios do sono e tiveram

Tabela 1 - Sintomas do Transtorno por Estresse Pós-Traumático

\begin{tabular}{c|c|c|c|c|c|c}
\hline \multirow{2}{*}{ SINTOMAS } & \multicolumn{2}{|c|}{$\begin{array}{c}\text { Sem História } \\
\text { de violência } \\
(\mathrm{n}=17)\end{array}$} & \multicolumn{2}{c|}{$\begin{array}{c}\text { Com História } \\
\text { de violência } \\
(\mathrm{n}=69)\end{array}$} & \multicolumn{2}{c}{$\begin{array}{c}\text { Violência na } \\
\text { gestação atual } \\
(\mathrm{n}=61)\end{array}$} \\
\cline { 2 - 9 } & $\mathrm{f}$ & $\%$ & $\mathrm{f}$ & $\%$ & $\mathrm{f}$ & $\%$ \\
\hline Sentimentos de desamparo e/ou impotência & 2 & 11,7 & 21 & 30,4 & 36 & 59,0 \\
\hline Falta de concentração & 6 & 35,2 & 30 & 43,4 & 34 & 55,7 \\
\hline Lapsos de memória & 2 & 11,7 & 19 & 27,5 & 29 & 47,5 \\
\hline Sonhos que se repetem. & 5 & 29,4 & 33 & 47,8 & 28 & 45,9 \\
\hline Flashbacks que fazem com que reviva o trauma & 6 & 35,2 & 46 & 66,6 & 51 & 83,6 \\
\hline Distúrbios do sono & 3 & 17,6 & 31 & 44,9 & 38 & 62,2 \\
\hline Assusta-se facilmente & 5 & 29,4 & 13 & 18,8 & 23 & 37,7 \\
\hline Dificuldade de orientação espacial & 1 & 5,8 & 10 & 14,4 & 17 & 27,8 \\
\hline Julga-se a si mesma / culpa & 7 & 41,1 & 32 & 46,3 & 51 & 83,6 \\
\hline Isolamento & 3 & 17,6 & 19 & 27,5 & 30 & 49,1 \\
\hline Diminuição do interesse pela vida & 0 & 0,0 & 4 & 5,7 & 9 & 14,7 \\
\hline Sente-se indigna / não se dá valor & 1 & 5,8 & 2 & 2,9 & 2 & 3,2 \\
\hline
\end{tabular}

sonhos que se repetiam, respectivamente. Nas mulheres em vivência de violência na gestação atual, este percentual sobe para $62,2 \%$ e $45,9 \%$, respectivamente.

Exceto no que se refere à categoria "sente-se indigna", os demais sentimentos tiveram seus percentuais consideravelmente aumentados em mulheres que vivenciaram violência na gestação atual na comparação com as mulheres sem histórias de violência, sugerindo que, embora o aborto esteja associado ao estresse pós-traumático, a vivência de violência potencializa os sintomas.

Mulheres que relatam histórico de trauma, tais como aborto ou abuso físico, estão mais sujeitas a apresentar sintomas do TEPT na comparação com mulheres sem história de abuso ${ }^{(17)}$. A violência doméstica durante a gestação pode trazer consequências, sobretudo para a saúde mental. As suas vítimas estão mais propensas a sofrer de estresse, depressão, consumir mais álcool e drogas e isso pode incluir a perda do interesse da mulher por sua saúde ${ }^{(4)}$.

Considera-se TEPT o transtorno de cunho emocional associado a um evento traumático ${ }^{(18)}$; a sensação de reviver o trauma gera angústia e sofrimento psicológico intensos, trazendo como consequências o isolamento social, a improdutividade profissional e a piora na qualidade de vida.

Born ${ }^{(17)}$ afirma que as mulheres possuem índices mais altos de comorbidade associadas ao TEPT e apresentam ansiedade, insônia, imagens em flashback, falta de concentração, comportamento evasivo, sonhos recorrentes e desinteresse. Podemos observar, de acordo com o estudo, que o aborto provocado traz consequências traumáticas para as mulheres, independentemente da vivência de violência doméstica. Contudo, nas mulheres com essa vivência, principalmente nas que sofreram violência na gestação, esses sintomas do TEPT são bem mais expressivos.

A natureza e a gravidade do trauma em mulheres constituem fatores de risco para o desenvolvimento do TEPT, o que torna as mulheres que sofreram violência na gestação, principalmente as que provocaram aborto em decorrência da violência, mais vulneráveis ao desencadeamento de problemas físicos e psicológicos ${ }^{(18)}$. Daí a necessidade de maior atenção por parte dos profissionais que assistem a mulheres em situação de aborto no sentido de melhor acolher a mulher, não apenas nas situações clínicas, mas também no que diz respeito à vivência de violência doméstica.

Os profissionais de saúde não podem restringir a assistência à esfera técnica. É preciso considerar as diversas demandas das mulheres ${ }^{(19)}$. Vale salientar a importância da investigação da temática, as notificações dos casos confirmados ou suspeitos, os protocolos de atendimento a muIheres em situação de abortamento, bem como os encaminhamentos à Rede de Atendimento às mulheres em situação de violência. 


\section{CONSIDERAÇÕES FINAIS}

As mulheres em processo de aborto provocado, sujeitos do estudo, foram principalmente mulheres jovens, negras e com baixo nível de escolaridade. Neste estudo, a situação de conjugalidade não interferiu na decisão de abortar, embora a idade e a escolaridade possam estar associadas à não utilização de medidas para a prevenção da gravidez, o que expõe as mulheres a uma gravidez indesejada e, por conseguinte, ao aborto inseguro e a suas complicações. A pouca idade foi o terceiro motivo mencionado pelas mulheres como justificativa para a prática do aborto.

Quase metade da amostra não tem qualquer fonte de renda e as demais exercem atividades de baixa remuneração, o que leva à dependência total ou parcial de companheiros ou de familiares. A situação econômica foi justamente o principal motivo alegado pelas mulheres para a realização do aborto. A segunda razão mais citada foi a vivência de violência doméstica.

Mais de um terço das entrevistadas já havia realizado pelo menos outro aborto, sinalizando para a necessidade de trabalhos educativos que valorizem o campo das subjetividades com adolescentes, mulheres e homens, que permitam desenvolver a consciência da vivência da sexualidade com entendimento do corpo fisiologicamente preparado para gestar. No entanto, a submissão da mulher ao homem a coloca em posição de aceitar sem questionar as vontades do companheiro, inclusive no que se refere às relações sexuais desprotegidas.

A maioria das entrevistadas disse ter história de violência doméstica, praticada pelo cônjuge e expressa nas formas psicológica, física e sexual. Quase metade das mulheres a vivenciaram durante a gravidez atual, $67 \%$ das quais declararam ser este o motivo que as levou a decidir pelo aborto. Resumindo, o estudo revela uma associação entre a vivência de violência doméstica e o aborto provocado. Também aponta para as repercussões do aborto provocado, sobretudo quando associado à vivência de violência, para a saúde mental das mulheres, que desenvolvem sintomatologias depressivas como o TEPT. Necessita-se, portanto, de um olhar por parte dos profissionais de saúde, de modo a dar a devida valorização a esta síndrome.

A identificação da vivência de violência doméstica pelos profissionais de saúde enquanto agravo associado ao aborto provado é de extrema importância para o processo de cuidar das mulheres, o que requer transformação no modelo de formação que incorpore a violência domestica como objeto da saúde e ações intersetoriais, especialmente no que tange aos encaminhamentos para serviços especializados no atendimento às pessoas em situação de violência.

\section{REFERÊNCIAS}

1. Organização Mundial da Saúde. International Women's Health Coalition. Abortamento Seguro: orientação técnica e política para os sistemas da saúde. Genebra: OMS; 2004.

2. Ministério da Saúde (BR). Secretaria de Ciência, Tecnologia e Insumos Estratégicos. Departamento de Ciência e Tecnologia. Aborto e saúde pública no Brasil: 20 anos. Brasília: Ministério da Saúde; 2009.

3. Loureiro DC, Vieira EM. Aborto: conhecimento e opinião de médicos dos serviços de emergência de Ribeirão Preto, São Paulo, Brasil, sobre aspectos éticos e legais. Cad. Saúde Pública. 2004 jun; 20 (3): 679-88.

4. Menezes TC, Amorim MMR, Santos LC, Faúndes A. Violência física doméstica e gestação: resultados de um inquérito no puerpério. Rev Bras Ginecol Obstet. 2003 jun; 25 (5): 309-16.

5. Ministério da Saúde (BR). Secretaria de Atenção à Saúde. Prevenção e Tratamento dos Agravos Resultantes da Violência Sexual contra Mulheres e Adolescentes: norma técnica. $2^{\mathrm{a}}$ ed. atual. e ampl. Brasília: Ministério da Saúde; 2005.

6. Ministério da Saúde (BR). Conselho Nacional de Saúde, Comissão Nacional de Ética em Pesquisa. Resolução $N^{\circ}$ 196 de 10 de outubro de 1996: aprova as diretrizes e normas regulamentadoras de pesquisa envolvendo seres humanos. Brasília: Ministério da Saúde; 1996.

7. American Psychiatric Association. Manual Diagnóstico e
Estatístico dos Transtornos Mentais (DSM-IV). 4 ed. Porto Alegre: Artes Médicas; 1994.

8. Martins IR, Costa SH, Freita SRS, Pinto CS. Aborto induzido em mulheres de baixa renda:dimensão de um problema. Cad. Saúde Pública. 1991 abr/jun; 7 (2): 251-66.

9. Harddy E, Rebello I, Faúndes A. Aborto entre alunas e funcionárias de uma universidade brasileira. Rev. Saúde Pública. 1993 abr; 27 (2):113-6.

10. Prefeitura Municipal de Salvador (BA). Secretaria Municipal de Saúde. Diagnóstico de saúde da população negra de Salvador. Bahia: Salvador; 2006.

11. Henringer R. Desiqualdades raciais no Brasil: síntese de indicadores e desafios no campo das políticas públicas. Cad Saúde Pública. 2002; 18 (Supl): 57-65.

12. Costa RG, Hardy E, Osis MJD, Faúndes A. A decisão de abortar: processo e sentimentos envolvidos. Cad Saúde Pública. 1995; 11 (1):97-105.

13. Yannoulas SC. Dossiê: Políticas Públicas e relações de gênero no mercado de trabalho. Brasília: CFEMEA; FIG/ CIDA; 2002.

14. Meshulam-Werebe D, Andrade MGO, Delouya D. Transtorno do estresse pós-traumático: enfoque psicanalítico. Rev. Bras. Psiquiatr. 2003; 25 (1 Supl): 37-40.

15. Diniz NMF, Lopes RLM, Arrazola LSD, Alves SLB, Almeida MS, Ferreira SL, et al. Violência doméstica e institucional nos serviços de saúde: experiência de mulheres. Rev Bras. Enferm. 2004 jun; 57 (1): 354-60. 
16. Fernandes SLSA. Violência doméstica na gestação e baixo peso ao nascer. [dissertação]. Salvador: Universidade Federal da Bahia; 2001.

17. Born L, Phillips SD, Steiner M, Soares CN. Trauma e ciclo reprodutivo feminino. Rev. Bras. Psiquiatr. 2005; 27 (2 Supl): $65-72$.
18. Ballone GJ. Transtorno por estresse pós-traumático. [on-line] 2004 [citado em: 2004 Abr 15]. Disponível em URL: < http://gballone.sites.uol.com.br/coce/postrauma.htlm $>$.

19. Rodrigues MML, Hoga LAK. Aborto espontâneo e provocado: sentimentos vivenciados pelos homens. Rev Bras. Enferm. 2006 jan/fev; 59(1): 14-19. 